\title{
Cd Recombination Laser in a Plasma Generated by an Electron Beam
}

\author{
J. J. ROCCA, H. L. MANCINI, AND B. WERNSMAN
}

\begin{abstract}
Laser action was obtained in the 1.40, 1.43, and $1.65 \mu \mathrm{m}$ lines of CdI following electron-ion recombination in a plasma generated by an electron beam. Lasing does not occur during the excitation pulse due to the electron impact population of the laser lower levels. In this plasma, supercooling of the electrons is achieved under stationary conditions, and efficient $\mathrm{CW}$ recombination laser action might also be possible on lines in which the lower level is not significantly populated by electron collisions. Seven new infrared laser lines in CdII are also reported.
\end{abstract}

$\mathrm{T}$ HE negative glow region of an electron-beam-created plasma, in which a large number of energetic beam electrons coexist with supercooled secondary electrons under stationary conditions, is shown to be an attractive medium to excite electron-ion recombination laser transitions.

We have obtained pulsed laser action in the $1.40,1.43$, and $1.65 \mu \mathrm{m}$ recombination lines of CdI. In addition, seven new laser lines were observed in CdII in the 1.1$2.0 \mu \mathrm{m}$ spectral region. It might also be possible to use electron beam pumping to obtain laser action at shorter wavelengths by electron-ion recombination in more highly ionized species.

The negative glow region of an electron-beam-created plasma has a unique electron energy distribution that presents advantages for the excitation of this type of laser transition as discussed hereafter. In the ideal recombination regime, the upper laser levels get populated at the rate of recombination of the immediately higher ionized specie $\mathrm{N}^{(i+1)+}$. In the dense and cold plasmas of interest for a recombination laser medium, three body electronion recombination, which favors the population of the higher levels, dominates over radiative recombination, and consequently, the pumping rate $P$ is

$$
P=\alpha_{i} \mathrm{~N}^{(i+1)+} n e^{2}[\mathrm{Te}]^{-9 / 2}
$$

where the coefficient $\alpha_{i}$ depends on the degree of ionization $i[1]$ and $n e$ is the electron density.

Thus, to achieve strong pumping of the laser upper levels, we need both a large density of energetic electrons to achieve a sufficiently high plasma density through intense

Manuscript received July 26, 1985; revised December 8, 1985. This work was supported by National Science Foundation Grant ECS-8404727 and the National Science Foundation-CONICET (U.S.-Argentina) International Program. The work of J. J. Rocca was supported by an NSF Presidential Young Investigator Award.

J. J. Rocca and B. Wernsman are with the Department of Electrical Engineering, Colorado State University, Fort Collins, CO 80523.

H. L. Mancini is with CITEFA-CONICET, Argentina.

IEEE Log Number 8407378. ionization and a low electron temperature $T e$. These are contradictory requirements for an ordinary steady-state discharge. However, both conditions coexist in an electron-beam-created plasma.

The use of a supercooled plasma generated by an electron beam to excite recombination laser transitions was first proposed by Gudzenko et al. [2]. As compared to other discharges having a thermalized distribution of electrons, the beam-sustained plasmas have a larger density of energetic electrons that can efficiently ionize. At the same time, supercooled electrons that readily recombine constitute the dominant group in the distribution. We have measured the electron energy of the group of cool electrons in a steady-state electron beam plasma obtaining values between 0.07 and $0.11 \mathrm{eV} \mathrm{[3]}$.

The low energy of these electrons results from the peak at zero energy of the differential cross section for the production of secondary electrons in ionizing collisions [4] and from the fact that there is essentially no electric field in the negative glow region of the electron-beam-created plasma. Consequently, the secondary electrons can only gain energy from superelastic collisions, elastic collisions with energetic electrons, and recombination processes. The large number of elastic electron-atom collisions keeps the electron temperature at an extremely low value. As a result, these plasmas are recombination dominated [5]. Since it is possible to deposit more than half of the electron beam energy into the creation of ions [6], rapid recombination of these ions can provide an efficient laser excitation mechanism.

To obtain the results reported here, we have used an experimental setup similar to the one previously employed to obtain laser action by charge transfer in $\mathrm{He}-$ metal vapor mixtures [6]. Pulsed excitation was used to demonstrate laser action in the $1.40,1.43$, and $1.65 \mu \mathrm{m}$ recombination transitions of $\mathrm{CdI}$ in an electron-beam-generated plasma. Electron beam pulses $(10-100 \mu \mathrm{s})$ of energy between 1 and $5 \mathrm{keV}$ and current up to $5 \mathrm{~A}$ were produced using a glow discharge electron gun having an aluminum cathode of novel design. The electron beam was confined by an axial magnetic field (1.5-3.5 kG) to allow efficient deposition of its energy in an $\mathrm{He}-\mathrm{Cd}$ mixture contained in a $1 \mathrm{~m}$ long plasma tube. The Cd vapor was produced by heating a reservoir to a temperature of $420^{\circ} \mathrm{C}$. The wall of the stainless steel plasma tube was kept $100^{\circ} \mathrm{C}$ above the reservoir temperature to avoid metal vapor condensation. Average plasma tube pressures between 2 and 4 torr were used.

When the device was provided with two internally mounted highly reflecting mirrors in the $1.0-1.5 \mu \mathrm{m}$ spec- 


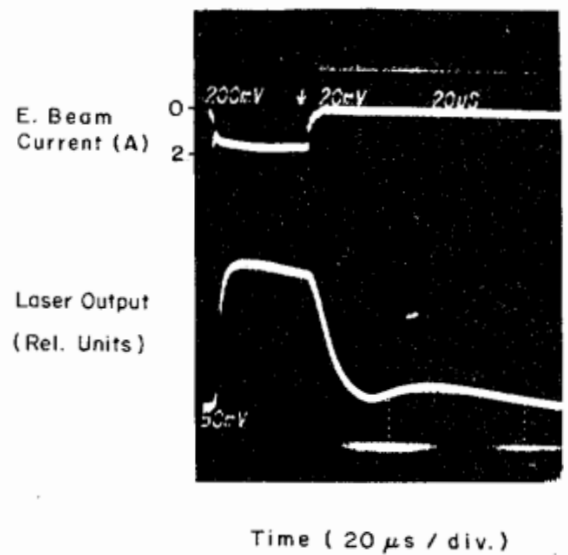

Fig. 1. Laser output (lower trace) and electron beam discharge current (upper trace) as a function of time. Increasing current is in a downward direction. The $\mathrm{Cd}$ reservoir temperature was $420^{\circ} \mathrm{C}$ and the magnetic field was $1.6 \mathrm{kG}$. The plasma tube average pressure was 4 torr.
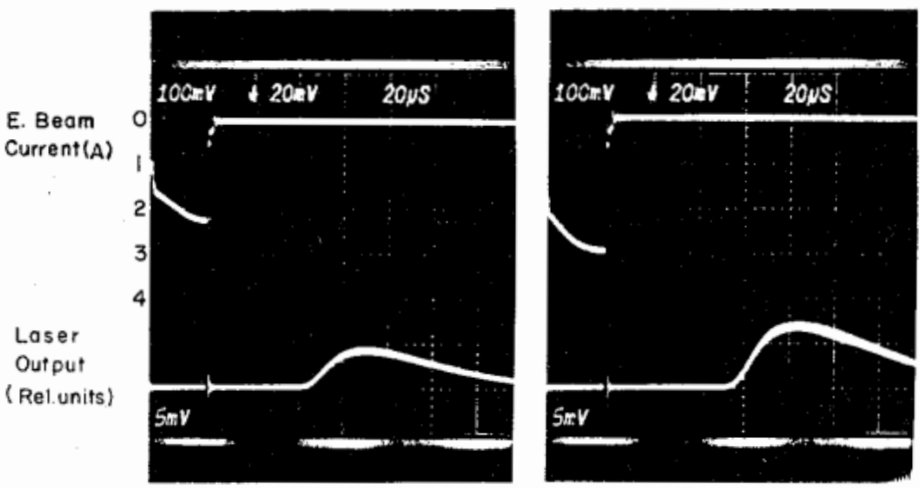

Time $(20 \mu \mathrm{s} /$ div. $)$

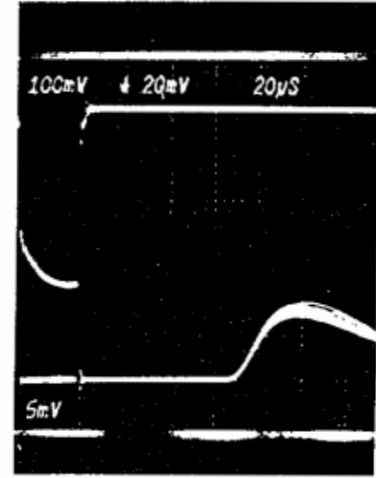

(2)

Fig. 2. $1.43 \mu \mathrm{m}$ laser output (lower trace) as a function of time for different electron beam discharge currents (upper trace). Increasing current is in a downward direction. The other parameters are as in caption of Fig. 1.

tral range, laser action was observed as illustrated by Fig. 1. The upper oscilloscope trace corresponds to the current pulse and the lower trace to the laser output as observed with a Ge photodiode. Lasing from the CdI $1.40 \mu \mathrm{m}(6 p$ $\left.{ }^{3} P_{2}^{0}-6 s^{3} S_{1}\right)$ and $1.43 \mu \mathrm{m}\left(6{ }^{3} P_{1}^{0}-6 s^{3} S_{1}\right)$ recombination lines occurs in the afterglow of the electron beam pulse with a delay of several tens of microseconds with respect to the termination of the electron beam pulse.

The delay increases as the intensity of the electron beam pulse increases. Fig. 2 shows that increasing the electron beam current pulse from 2 to $4 \mathrm{~A}$ increases the delay from 44 to $70 \mu \mathrm{s}$. The variation of the peak intensity of the 1.43 $\mu \mathrm{m}$ laser pulse as a function of electron beam current is illustrated in Fig. 3. The increase of the time delay of the onset of the laser pulse and the saturation of the peak intensity with increasing current are due to superelastic electron de-excitation of the laser upper level.

Figs. 2 and 3 show that increasing the electron beam current above $3 \mathrm{~A}$ does not significantly increase the intensity of the laser pulse and results only in an increased delay. This occurs because after the termination of the electron beam pulse the electron density has to fall below a certain critical value for a significant population inversion to be established. A similar delay was previously observed in recombination lasers in spark discharges [7], and a criterion was developed to calculate the critical electron density above which inversions are reduced by electron collisions [8]. Electron de-excitation limits the laser energy density that can be extracted from the CdI recombination lines to a low value $\left(<10^{-6} \mathrm{~J} / \mathrm{cm}^{3}\right)$. No attempt was made to optimize the laser cavity to obtain the maximum possible laser power. Nevertheless, at shorter wavelengths, significant electron de-excitation occurs only at higher plasma densities [8]; consequently, the excitation technique demonstrated here could prove useful to efficiently pump lasers generating considerably higher energy outputs.

However, an excessive electron density is not the only reason for the delay shown in Fig. 2. By reducing the excitation current, it is not possible to avoid a delay. Electron impact excitation of the lower laser levels is judged to be the reason for which laser action is not ob- 


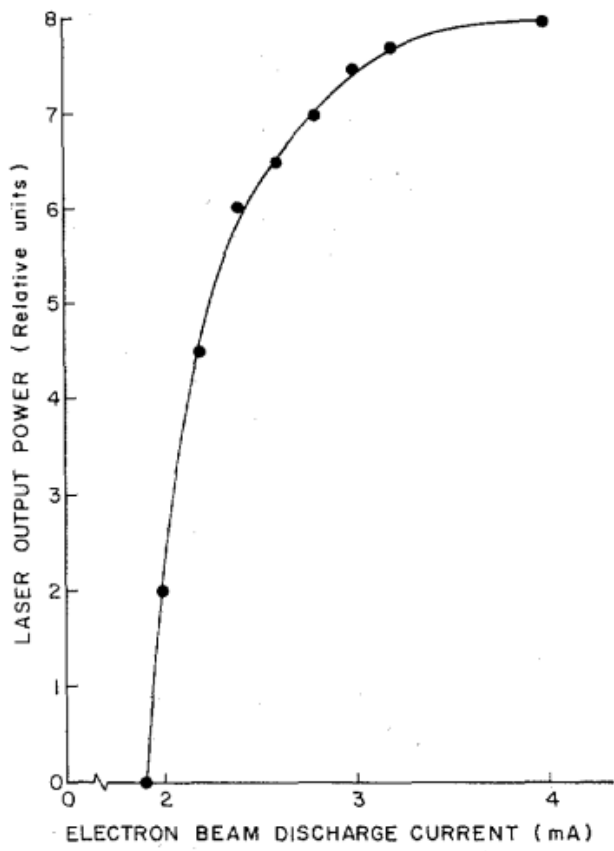

Fig. 3. $1.43 \mu \mathrm{m}$ peak laser power output as a function of electron beam discharge current. Electron beam pulse width: $30 \mu \mathrm{s}$. Cd reservoir temperature: $420^{\circ} \mathrm{C}$. Magnetic field: $2.1 \mathrm{kG}$. The plasma tube average pressure was 4 torr.

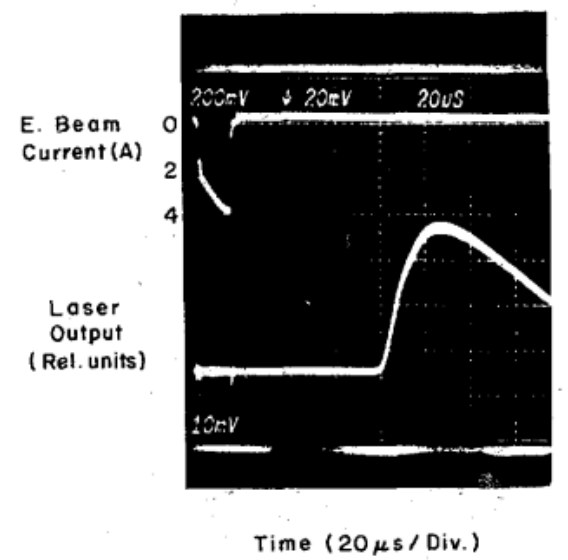

Fig. 4. Time dependence of the $1.65 \mu \mathrm{m}$ laser output (lower trace). The upper trace corresponds to the electron beam current pulse. Increasing current is in a downward direction.

tained during or immediately after the excitation pulse. In contrast to other plasmas, the electron-beam-created plasma is supercooled at stationary conditions, and in the absence of significant electron impact excitation of the laser lower level, $\mathrm{CW}$ laser action should be expected. This, however, does not occur in CdI. Electrons having enough energy to excite the laser lower levels have to thermalize before a significant inversion can be established.

When mirrors having high reflectivity in the 1.5-2.0 $\mu \mathrm{m}$ spectral region were used, laser output was obtained in the CdI $1.65 \mu \mathrm{m}\left(4 f^{3} F_{4}^{0}-5 d^{3} D_{3}\right)$ line as shown in Fig. 4. This transition presents, as expected, a behavior similar to the other two recombination lines.

The laser output occurring during the excitation pulse

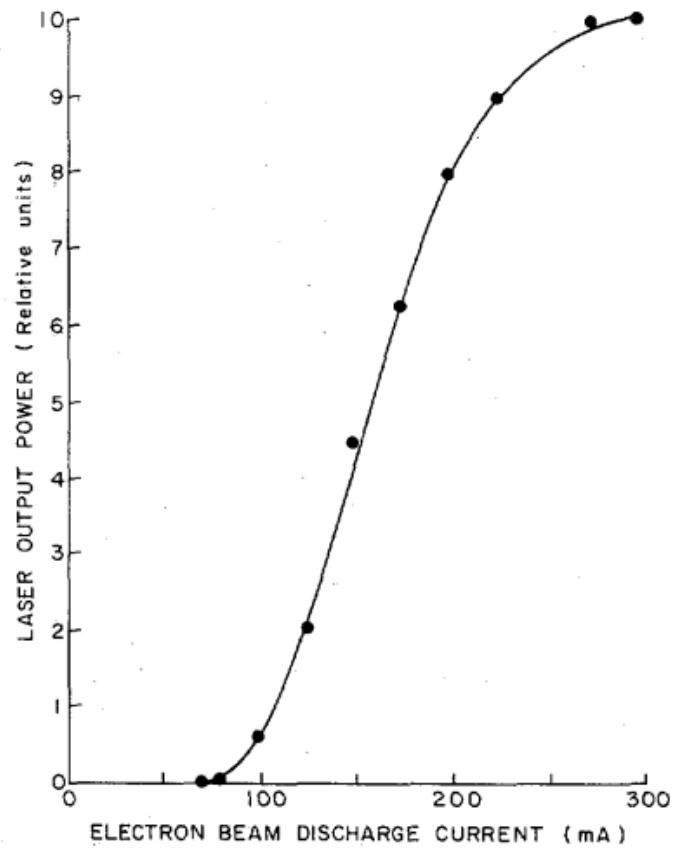

Fig. 5. CW laser output power of the $1.85 \mu \mathrm{m}$ line as a function of electron beam discharge current and voltage. The magnetic field was $2.9 \mathrm{kG}$ and the plasma tube average pressure was 2 torr.

in Fig. 1 was measured to be at $(18498.8 \pm 2) \AA$. Laser action occurred only at $\mathrm{Cd}$ reservoir temperatures above $365^{\circ} \mathrm{C}$. In Fig. 1, the $1.85 \mu \mathrm{m}$ line is observed to oscillate despite the mirrors having only 39 percent reflectivity at that wavelength. By mounting one of the mirrors externally and introducing calibrated losses into the cavity, we measured a gain coefficient of 3 percent $\cdot \mathrm{cm}^{-1}$ for this transition. When direct current electron beam excitation was provided, $\mathrm{CW}$ laser oscillation was observed at 1.85 $\mu \mathrm{m}$. The current threshold for $\mathrm{CW}$ oscillation was $70 \mathrm{~mA}$. Fig. 5 shows the variation of the $\mathrm{CW}$ laser output of this line with electron beam current. None of the known impurities present in the plasma has a transition at this wavelength. This line could be possibly assigned to the $18500.7 \AA\left(5 p^{2}{ }^{3} P_{1}-9 p^{3} P_{2}^{0}\right)$ transition of CdI.

Excitation of the $5 p^{2}{ }^{3} P_{1}$ level by electron impact has been previously observed [9]. Doubly excited levels are usually autoionized with a high probability [10] and, consequently, are not good laser upper levels. However, the ${ }^{3} P_{1}$ is not subject to autoionization because of the selection rules [10]. A drawback to such an assignment is the fact that the transition probability from the level $5 p^{2}$ to $9 p$, and thus the gain on this line, would be expected to be very low since it is a double electron transition. Also, it is not clear why the $9 p$ level would be favored over the other $n p$ levels. A more detailed investigation is needed to unambiguously assign the $1.85 \mu \mathrm{m}$ line.

In addition to the $1.85 \mu \mathrm{m}$ line and the three $\mathrm{CdI}$ recombination lines, we observed seven new laser transitions in CdII at wavelengths between 1.1 and $2.0 \mu \mathrm{m}$. The wavelengths, levels assigned, and threshold currents for $\mathrm{CW}$ oscillation are included in Table I. The only new CdII laser transition not observed in continuous wave operation was the $1.19450 \mu \mathrm{m}$ line. The laser upper levels of some 
TABLE I

Observed Laser Transitions

\begin{tabular}{cllc}
\hline \hline $\begin{array}{c}\text { Wavelength } \\
(\mu \mathrm{m})\end{array}$ & Spectrum & Transition Assignment & $\begin{array}{c}\text { CW Current Threshold } \\
(\boldsymbol{A})\end{array}$ \\
\hline $1.19450^{a}$ & $\mathrm{Cd} \mathrm{II}$ & $5 f^{2} F_{5 / 2}^{0}-6 d^{2} D_{3 / 2}$ & - \\
$1.21999^{a}$ & $\mathrm{Cd} \mathrm{Il}$ & $5 f^{2} F_{7 / 2}^{0}-6 d^{2} D_{5 / 2}$ & 0.2 \\
1.39827 & Cd I & $6 p^{3} P_{2}^{0}-6 s^{3} S_{1}$ & - \\
1.43316 & Cd I & $6 p^{3} P_{1}^{0}-6 s^{3} S_{1}$ & - \\
1.64862 & Cd I & $4 f^{3} F_{4}^{0}-5 d^{3} D_{3}$ & - \\
$1.75226^{a}$ & Cd II & $6 g^{2} G_{9 / 2}-5 f^{2} F_{7 / 2}^{0}$ & - \\
$1.78396^{a}$ & Cd II & $6 g^{2} G_{7 / 2}-5 f^{2} F_{5 / 2}^{0}$ & 1.1 \\
$1.80510^{a}$ & Cd II & $6 p^{2} P_{3 / 2}^{0}-5 d^{2} D_{5 / 2}$ & 0.28 \\
$1.84988^{a, b}$ & Cd I $(?)$ & $5 p^{2}{ }^{3} P_{1}-9 p^{3} P_{2}^{0}(?)$ & 0.07 \\
$1.92696^{a}$ & Cd II & $7 p^{2} P_{3 / 2}^{0}-7 s^{2} S_{1 / 2}$ & 0.58 \\
$1.99157^{a}$ & Cd II & $6 p^{2} P_{1 / 2}^{0}-5 d^{2} D_{3 / 2}$ & 0.70 \\
\hline
\end{tabular}

${ }^{a}$ New laser line.

${ }^{b}$ Measured wavelength.

of these transitions $\left(6 g^{2} G\right)$ are in close proximity with the energy of the helium ion ground state. Consequently, they are expected to be dominantly populated by charge transfer collisions between ground state helium ions and cadmium atoms [11]. Radiative cascade from levels populated by charge transfer is also judged to make a significant contribution to the population of the upper levels of the remaining CdII laser transitions. As expected, laser radiation was achieved in the 5337 and $5378 \AA$ lines of CdII when the infrared mirrors were replaced by mirrors reflecting in the green.

Summarizing, laser action has been obtained in three CdI transitions following electron-ion recombination in an electron-beam-generated plasma. Oscillation was also obtained on seven new transitions in CdII. Electron beams can be used to generate stationary plasmas that are simultaneously dense and cold. Supercooling of the electrons is accomplished under steady-state conditions, and CW recombination laser action might result in laser systems in which the lower level is not significantly populated by electron collisions. It should be possible to extend the technique used in this work to higher ionization stages to obtain efficient laser action in the visible and UV spectral regions.

\section{REFERENCES}

[1] V. Zhukov, E. Latush, V. Mikhalewskii, and S. Sem, "Recombination lasers using vapors of chemical elements,"'Sov. J. Quantum Electron, vol. 7, pp. 704-713, 1977.

[2] L. Gudzenko, M. Nezuin, and S. Yakovienko, "Recombination laser with a supercooled plasma generated by an electron beam," Sov. Phys. Tech. Phys., vol. 18, pp. 1218-1221, 1974.

[3] Z. Yu, J. J. Rocca, G. J. Collins, and C. Y. She, "The energy of thermal electrons in electron beam created helium discharges," Phys. Lett., vol. 96A, pp. 125-128, 1983.

[4] L. R. Peterson, "Discrete deposition of energy by electrons in gases," Phys. Rev., vol. 187, pp. 105-111, 1969.

[5] K. B. Persson, "Brush cathode plasma-A well behaved plasma," $J$. Appl. Phys., vol. 36, pp. 3086-3094, 1965.

[6] J. J. Rocca, J. Meyer, and G. J. Collins, "1-W CW Zn ion laser," Appl. Phys. Lett., vol. 43, pp. 37-39, 1983. See also J. J. Rocca, "Electron beam pumping of $\mathrm{CW}$ ion lasers," $\mathrm{Ph}$. D. dissertation, Colorado State Univ., Fort Collins.

[7] W. Silfvast, L. Szeto, and O. Wood, "Simple metal-vapor recombination lasers using segmented plasma excitation," Appl. Phys. Lett., vol. 36, pp. 615-617, 1980 .

[8] O. Wood and W. Silfvast, "Electron density and energy output limits of plasma recombination lasers," Appl. Phys. Lett., vol. 41, pp. 121123,1982

[9] A. Ruark, "Multiple electron transitions and primed spectral terms," J. Opt. Soc. Amer. and Rev. Sci. Instrum., vol. 1, pp. 199-212, 1925.

[10] E. V. Condon and G. H. Shortley, The Theory of Atomic Spectra. Cambridge, England: Cambridge Univ. Press, 1959.

[11] A. Turner-Smith, J. Green, and C. Webb, "Charge transfer into excited states in thermal energy collisions," J. Phys. B: Atom. Molec. Phys., vol. 6, pp. 114-130, 1973. 